\title{
PEGylated Red-Emitting Calcium Probe with Improved Sens- ing Properties for Neuroscience
}

\author{
Flavien Ponsot, ${ }^{\ddagger 1}$ Weida Shen,${ }^{\$ 2,3}$ Pichandi Ashokkumar, ${ }^{1}$ Etienne Audinat, ${ }^{2,3}$ Andrey S. Klym- \\ chenko, ${ }^{1}$ Mayeul Collot ${ }^{1 *}$ \\ ${ }^{1}$ Laboratoire de Biophotonique et Pharmacologie, UMR 7213 CNRS, Université de Strasbourg, Faculté de Pharmacie, 74, \\ Route du Rhin, 67401, Illkirch, France. \\ ${ }^{2}$ INSERM, U1128, Paris, France \\ ${ }^{3}$ Laboratory of Neurophysiology and New Microscopies, Paris Descartes University, Paris, France
}

KEYWORDS : Fluorescent calcium probes, PEGylation, compartmentalization, dendritic calcium signal, neuron.

\begin{abstract}
Monitoring calcium concentration in the cytosol is of main importance as this ion drives many biological cascades within the cell. To this end, molecular calcium probes are widely used. Most of them, especially the red emitting probes suffer from non-specific interactions with inner membranes due to the hydrophobic nature of their fluorophore. To circumvent this issue, calcium probes conjugated to dextran can be used to enhance the hydrophilicity and reduce the non-specific interaction and compartmentalization. However, dextran conjugates also feature important drawbacks including lower affinity, lower dynamic range and slow diffusion. Herein, we combined the advantage of molecular probes and dextran conjugate without their drawbacks by designing a new red emitting turn-on calcium probe based on PET quenching, Rhod-PEG, in which the rhodamine fluorophore bears four $\mathrm{PEG}_{4}$ units. This modification led to a high affinity calcium probe $(\mathrm{Kd}=748$ $\mathrm{nM}$ ) with reduced non-specific interactions, enhanced photostability,two-photon absorbance and brightness compared to the commercially available Rhod-2. After spectral characterizations, we showed that Rhod-PEG quickly and efficiently diffused through the dendrites of pyramidal neurons with an enhanced sensitivity $\left(\Delta \mathrm{F} / \mathrm{F}_{0}\right)$ at shorter time after patching compared to Rhod-2.
\end{abstract}

Calcium ions $\left(\mathrm{Ca}^{2+}\right)$ are ubiquitous second messenger involved in many functions and processes of cells including proliferation, gene regulation or cell death. ${ }^{1}$ In neurons, $\mathrm{Ca}^{2+}$ regulates multiple aspects of its function, participates in the transmission of the depolarizing signal and contributes to synaptic activity. ${ }^{2}$ Therefore monitoring intracellular calcium concentration $\left[\mathrm{Ca}^{2+}\right]_{\mathrm{i}}$ is of prior importance in order to better understand the cellular and more specifically, neuronal mechanisms. ${ }^{3,4}$ The fast development of fluorescence imaging technics and microscopies has led the chemists to developing fluorescent calcium probes. ${ }^{5}$ Although the first fluorescent calcium probes were developed in the eighties by Tsien and colleagues, ${ }^{7}$ continuous progresses have been made in the field by improving several parameters responding to certain demands. Recently, efficient genetically encoded fluorescent calcium indicators have been proposed in the literature, $8,9,10$ nevertheless small molecular calcium probes remain valuable tools in calcium imaging as they do not require any transfection step and are more user-friendly. The widespread use of green or yellow fluorescent proteins in cell biology has drawn the demand towards red shifted calcium probes thus allowing multicolour imaging. ${ }^{10,11,12,13,14}$ For these rea- sons red-emitting rhodamine-based $\mathrm{Ca}^{2+}$ probes like Rhod$2^{15}$ were developed. Nevertheless, unlike green emitting fluorescein-based ones, they tend to compartmentalize in inner membranes and organelles due to the hydrophobic and cationic nature of the fluorophore. ${ }^{16}$ Recently a siliconfluorescein based calcium probe was introduced with reduced compartmentalization by combining the hydrophilic nature of a fluorescein and the red shift emission provoked by the silicon atom. ${ }^{11,16}$ Most commonly, in order to circumvent the issue of compartmentalization, dextran, a hydrophilic polysaccharide, is conjugated to the calcium probe to reduce the hydrophobic interactions. ${ }^{15,17}$ However, dextran conjugates suffer from several drawbacks: 1) The affinity of the conjugated probe is reduced compared to the parent free probe due to the use of one of its carboxylate group for conjugation to amino-dextran. This problem was addressed by the introduction of the red emitting calcium probes family: Calcium Rubies. ${ }^{18,19,20}$ These probes display various affinity (from $20 \mu \mathrm{M}$ to $258 \mathrm{nM}$ ) and have the distinctive feature to possess an azido side-arm that served for conjugation to dextran using the CuAAC orthogonal click chemistry without affecting the affinity. 2) Even though low molecular weight dextran are available, after 
functionalization with calcium probes the high molecular weight of the conjugates limit their diffusion speed within the cytosol of the neuron. 3) The homogeneity of the batch is not guaranteed, as it is difficult to control the number of probes attached per dextran molecule. 4) The brightness as well as the fluorescence enhancement are reduced due to the quenching phenomenon. Indeed concentrating several fluorophores on the same dextran molecule favors the formation of $H$-aggregates which produces non-emissive dark species. $^{21}$ This phenomenon is identified by the increase of the blue shifted shoulder in the absorbance spectrum. ${ }^{19,22}$ As far as our knowledge, the last three points have not yet been addressed.

Herein we developed a red emitting turn-on calcium probe based on a rhodamine fluorophore that bears four $\mathrm{PEG}_{4}$ units. From this design, arose an improved version of Rhod-2 probe with greatly reduced non-specific interactions and compartmentalization and thus allowed for monitoring calcium transients further in the dendrites, in a faster manner and with an enhanced sensitivity. Rhod-PEG combines the advantage of dextran conjugates in terms of hydrophilicity and reduced compartmentalization with the homogeneity, small size and brightness of small molecular calcium probes.

\section{RESULTS AND DISCUSSION}

\section{Synthesis of Rhod-PEG}

Red emitting calcium probes are generally composed of a calcium chelating moiety, mostly BAPTA ((1,2-bis- $(o-$ aminophenoxy)ethane- $N, N, N^{\prime}, N^{\prime}$-tetraacetic acid)) and a red-emitting fluorophore. At the OFF state the electron rich hydroxyanilin of the BAPTA turns off the fluorophore through the PET (photoinduced electron transfer) phenomenon. Upon binding of the calcium ion to the BAPTA, the PET quenching is blocked, revealing the fluorescence of the sensor. Among these red-emitting calcium probes, Rhod-2 is a widely used commercially available one, which is composed of a BAPTA and a tetramethyl rhodamine (Figure 1).
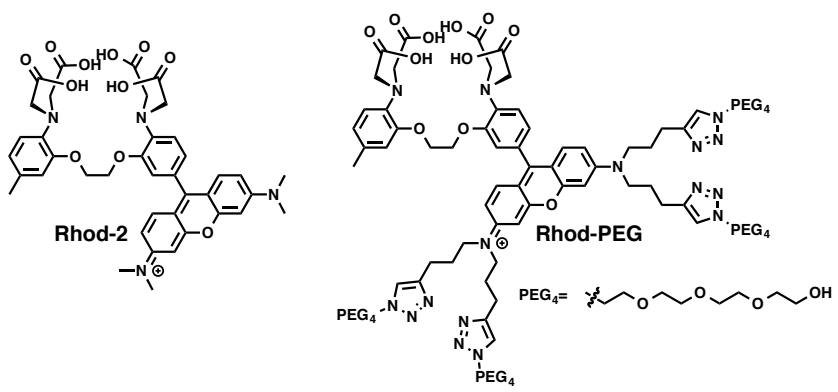

Figure 1. Chemical structures of Rhod-2 and Rhod-PEG.

Owing to the hydrophobic nature of the fluorophore, Rhod-2 tends to quickly penetrate the cells and to compartimentalize in inner membranes. Polyethylene glycol is a hydrophilic and polar molecule known to reduce the nonspecific interaction with biomolecules in both nanoparti$\operatorname{cles}^{23,24}$ and molecular sensors. ${ }^{25}{ }^{26}$ Although the PEGyla- tion of a green emitting calcium probe based on a BODIPY fluorophore has recently been described to enhance the cellular uptake, ${ }^{27}$ we herein postulate that a PEGylated rhodamine-based $\mathrm{Ca}^{2+}$ probe should display a reduced cell permeability as well as avoid compartmentalization when patched in a neuron, thus allowing enhanced diffusion.

Due to the symmetry of rhodamine fluorophore, adding a single side arm for further functionalization to dextran or PEG can be tedious in term of synthesis. ${ }^{18,}{ }^{19}$ Herein we modified the rhodamine fluorophore with 4-pentynyl groups leading to molecule 3 thus allowing for further modifications by $\mathrm{CuAAC}$ click chemistry (scheme 1). Dye 3 was then clicked with four $\mathrm{PEG}_{4}$ groups and the carboxylic groups were then deprotected to provide Rhod-PEG in a pure form after reverse phase column chromatography.

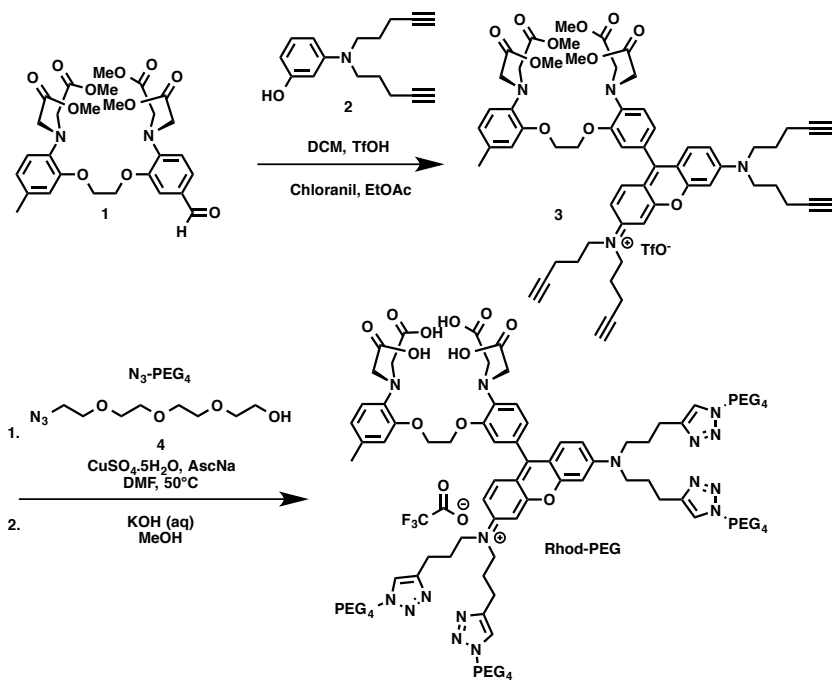

Scheme 1. Synthesis of Rhod-PEG.

\section{Spectroscopy}

First, the water solubility of Rhod-PEG was checked and it appeared to be soluble at high concentrations ( $\mathrm{mM}$ range) thus proving the efficiency of the four PEG chains in enhancing the hydrophilicity of the fluorophore. It is an important property for patching experiments as calcium sensors are dissolved at high concentrations in high ionic strength aqueous solution that can sometimes lead to pipette clogging. The photo-physical properties of Rhod-PEG were then evaluated. It is noteworthy that whereas minor chemical modifications have been brought to the fluorophore, Rhod-PEG exhibits different spectral characteristics from Rhod-2 (Table 1).

Although Rhod-PEG showed similar molar extinction coefficient $\left(82,000 \mathrm{~cm}^{-1} \mathrm{M}^{-1}\right)$, it was slightly red-shifted compared to Rhod-2. Indeed, Rhod-PEG displayed a maximum absorption wavelength at $562 \mathrm{~nm}$ and maximum emission wavelength at $587 \mathrm{~nm}$, which would allow, during microscopy experiments, more efficient excitation (using a widely used $560 \mathrm{~nm}$ laser) and a larger amount of collected photons, respectively. Upon titration with increasing concentrations of free $\mathrm{Ca}^{2+}$, Rhod-PEG displayed typical PET quenching calcium probe behavior where the absorption spectra were virtually unchanged whereas the fluorescence intensity increased (Figure $2 \mathrm{~A}$ and B). Importantly, Rhod- 
PEG exhibited an impressive 200-fold fluorescence enhancement.

Table 1. Photo- and physico-chemical properties of Rhod-PEG and Rhod-2. Concentration of sensors was $1 \mu \mathrm{M}$ in MOPS (30 mM), $\mathrm{KCl}(100 \mathrm{mM}), \mathrm{pH}$ 7.2. $\mathrm{Ca}^{2+}$ free is $1 \mathrm{mM}$ EGTA, and $\mathrm{Ca}^{2+}$ bound is $1 \mathrm{mM} \mathrm{Ca}^{2+}$.

\begin{tabular}{ccccccc}
\hline Probe & $\begin{array}{c}\lambda_{\text {Abs max }} / \lambda_{\text {Em max }} \\
\left(\mathrm{Ca}^{2+} \text { free }\right)\end{array}$ & $\begin{array}{c}\lambda_{\text {Abs max }} / \lambda_{\text {Em max }} \\
\left(\mathrm{Ca}^{2+} \text { bound }\right)\end{array}$ & $\begin{array}{c}\varepsilon_{\max } \\
\left(\mathbf{c m}^{-1} \mathbf{M}^{-1}\right)\end{array}$ & $\begin{array}{c}\text { Kd } \\
(\mathrm{nM})\end{array}$ & $\begin{array}{c}\text { Fluorescence } \\
\text { enhancement }\end{array}$ & $\begin{array}{c}\text { QY (\%) } \\
\text { OFF/ON }\end{array}$ \\
\hline Rhod-2 & $549 /$ None $^{\mathrm{a}}$ & $552 / 581^{\mathrm{a}}$ & $82,000^{\mathrm{a}}$ & $570^{\mathrm{a}}$ & $>100-$ fold $^{\mathrm{a}}$ & $0.03 / 10.2$ \\
Rhod-PEG & $558 / 583$ & $562 / 587$ & 82,000 & 748 & 200 -fold & $0.06 / 24^{\mathrm{b}}$
\end{tabular}

${ }^{a}$ data given by thermofisher. ${ }^{15}$

${ }^{\mathrm{b}}$ The quantum yield was measured with Rhodamine 101 as a reference $\left(\mathrm{QY}=100 \%\right.$ in EtOH). ${ }^{29}$

Compared to Rhod-2, Rhod-PEG also benefited from a low quantum yield in calcium free conditions $(\mathrm{QY}=0.06 \%)$ but displayed a higher one in calcium-bound form $(\mathrm{QY}=$ $24 \%$ ), thus conferring Rhod-PEG an impressive 400-fold quantum yield fluorescence enhancement upon calcium binding. Titration curves fitted with Hill's equation provided a slightly higher dissociation constant than Rhod-2 of $748 \pm 12 \mathrm{nM}$ (Figure 2C). Since magnesium $\left(\mathrm{Mg}^{2+}\right)$ is present at high concentration in the cytosol of cells, we checked whether $\mathrm{Mg}^{2+}$ could interfere with the $\mathrm{Ca}^{2+}$ sensing ability of Rhod-PEG. Figure 2D shows that high concentrations of $\mathrm{Mg}^{2+}$ does not provoke a significant fluorescence enhancement as well as does not disturb the $\mathrm{Ca}^{2+}$ sensing of Rhod-PEG (Figure 2D).
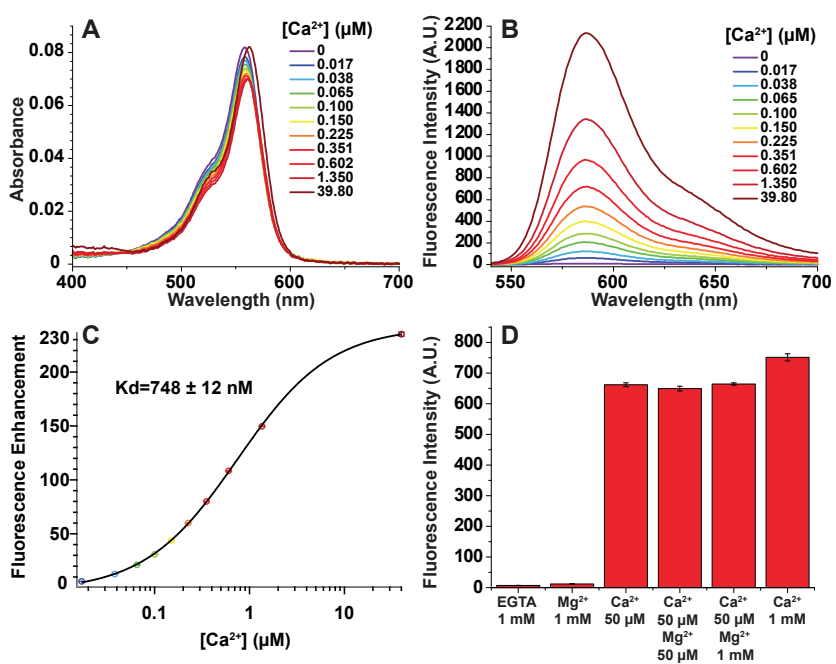

Figure 2. Absorption (A) and emission (B) spectra of Rhod-PEG $(1 \mu \mathrm{M})$ upon titration with increasing concentration of calcium, 0 (EGTA $1 \mathrm{mM}$ ) to $39 \mu \mathrm{M} \mathrm{Ca}^{2+}$ (in $30 \mathrm{mM}$ MOPS buffer, $100 \mathrm{mM}$ $\mathrm{KCl}, \mathrm{pH}$ 7.2). (C) Titration curves and the fit, according to Hill's equation, yielding the $\mathrm{Kd}$. Fluorescence enhancement is $\Delta \mathrm{F} / \mathrm{F}_{0}$ $\left(\mathrm{F}_{0}=\right.$ EGTA $\left.1 \mathrm{mM}\right)$. Each point represents the average of three independent experiments. (D) Influence of $\mathrm{Mg}^{2+}$ on the $\mathrm{Ca}^{2+}$ sensing ability of Rhod-PEG $(1 \mu \mathrm{M})$. Excitation wavelength was at $530 \mathrm{~nm}$.
Photodegradation experiment of Rhod-PEG was also performed and compared to Rhod-2 (Figure S1). Both sensors proved to be robust as Rhod-PEG displayed no loss of fluorescence intensity, whereas Rhod-2 lost only $20 \%$ of its intensity after 1-hour irradiation.

We then checked whether a $\mathrm{pH}$ variation could interfere with the calcium probing of Rhod-PEG. Titration with solution of various $\mathrm{pH}$ showed a 1.6-fold decrease in fluorescence intensity upon acidification, which is insignificant compared to the 200-fold increase upon calcium binding (Figure S2). Moreover, titration curve fitted with Hill's equation provided a $\mathrm{pKa}$ value of $6.27 \pm 0.03$ which is significantly lower than in the $\mathrm{pH}$-buffered cytosol. Never the less, the ability of Rhod-PEG to sense $\left[\mathrm{Ca}^{2+}\right]$ variation was checked at $\mathrm{pH} 4$ as crosstalk read-out can occur. ${ }^{30}$ The results showed that the fluorescence enhancement was dramatically decreased to 7.6-fold (Figure S3). This could be problematic for sensing $\left[\mathrm{Ca}^{2+}\right]$ variations in endosomes or lysosomes, however Rhod-PEG was designed to diffuse freely in the cytosol by patching and therefore it should not follow the endosomal pathway.

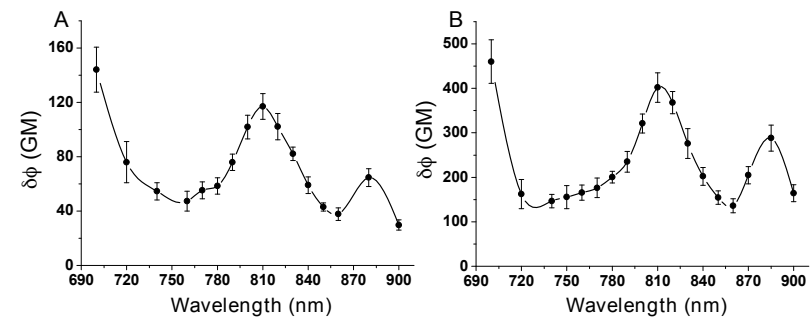

Figure 3. Two-photon excitation action cross-section spectra of Rhod-2 (A) and Rhod-PEG (B) in the presence of $1 \mathrm{mM} \mathrm{Ca}^{2+}$ (in $30 \mathrm{mM}$ MOPS, $100 \mathrm{mM} \mathrm{KCl}, \mathrm{pH}$ 7.2). The reference used for measurements was rhodamine B. GM is Goeppert-Mayer unit.

Finally the two-photon absorption spectra of Rhod-2 and Rhod-PEG were measured and compared. Indeed, in neuroscience, patch clamp experiments are performed in brain slices at several $\mu \mathrm{m}$ depth, thus for better light penetration two-photon excitation imaging is required. The results showed that at $810 \mathrm{~nm}$, Rhod-PEG benefit from an absorbance cross-section value $(\delta)$ increased by $46 \%$ compared to Rhod 2 (Figure S4). Consequently, due to its higher quan- 
tum yield value compared to Rhod-2, Rhod-PEG displayed significant higher action cross-section values $(\delta \phi)$ and make it more suitable for two-photon excitation imaging (Figure 3).

\section{Non-specific interactions}

Encouraged by these results, we then investigated on the ability of Rhod-PEG in avoiding non-specific interactions with membranes. To this endeavor, Rhod- 2 and Rhod-PEG $(1 \mu \mathrm{M})$ were placed in the presence of an excess of liposomes $(200 \mu \mathrm{M}$ of lipids) of different composition. The results showed that although Rhod-2 displayed an important fluorescence enhancement (between 3.6 and 4 fold) denoting interaction with membranes corresponding to hydrophobic interactions between the fluorophore and the lipids, Rhod-PEG displayed only very weak fluorescence intensity variations (Figure 4). These results tend to prove that the PEGylation of the fluorophore considerably reduce its affinity towards the membranes.

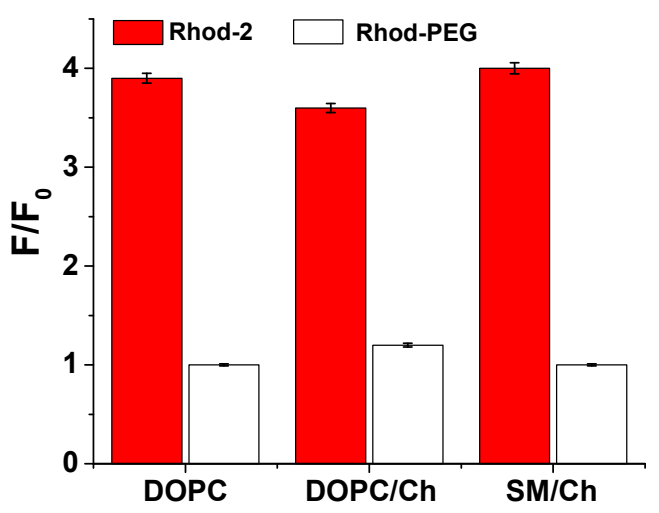

Figure 4. Fluorescence enhancement $\left(\mathrm{F} / \mathrm{F}_{0}, \mathrm{~F}_{0}=\right.$ fluorescence intensity in phosphate buffer, $20 \mathrm{mM}, \mathrm{pH} 7.4)$ of Rhod-2 $(1 \mu \mathrm{M})$ and Rhod-PEG $(1 \mu \mathrm{M})$ in the presence of various compositions of liposomes $(200 \mu \mathrm{M})$ in phosphate buffer $(20 \mathrm{mM}, \mathrm{pH}$ 7.4). DOPC: 1,2-Dioleoyl-sn-glycero-3-phosphocholine; Ch: Cholesterol; SM : Sphingomyelin. DOPC/Ch (1 :0.9), SM/Ch (1:0.9). Excitation wavelength was at $530 \mathrm{~nm}$.

In a next experiment, Rhod-2 and Rhod-PEG were placed at the same concentration $(1 \mu \mathrm{M})$ in the presence of $\mathrm{KB}$ cells (sub-line of the tumour cell line HeLa), which were directly imaged by laser scanning confocal microscopy. First, it is noteworthy that Rhod-PEG displayed a more intense fluorescence in the $\mathrm{Ca}^{2+}$ rich extracellular media compared to Rhod-2 (Figure 5A and 5D). This difference can be attributed to its higher quantum yield value as well as its $10 \mathrm{~nm}$ red shifted excitation spectrum that enhances its excitation efficacy. The images showed that whereas Rhod-2 was used instead of its cell permeable version based on acetoxymethyl esters (Rhod-2-AM), it immediately penetrates the cells and stain mitochondria ${ }^{16}$ (Figure S5), Rhod-PEG displays a bright fluorescence in the medium leaving the cytoplasm unstained. Incubation for two hours in the presence of $1 \mu \mathrm{M}$ of probes followed by washing steps led to the same results (Figure 5B and 5E). This comparison tends to show that the PEGylation of the fluorophore prevents its interaction with the plasma membrane and therefore avoid its penetration in the cytosol.

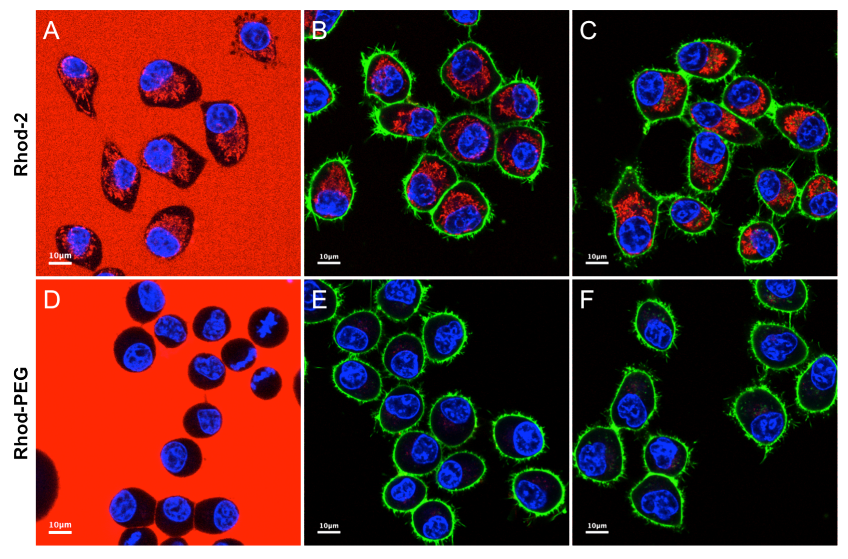

Figure 5. Laser scanning confocal microscopy images of KB cells in the presence of (A) Rhod-2 and (D) Rhod-PEG $(1 \mu \mathrm{M})$ directly after addition and without any washing step. Images of KB cells incubated in opti-MEM with (B) Rhod-2 and (E) Rhod-PEG (1 $\mu \mathrm{M}$ ) for 2 hours at $37^{\circ} \mathrm{C}$, the cells were washed two times with HBSS and visualized in HBSS. (C-F) ATP $(100 \mu \mathrm{M})$ was used to reveal the presence of Rhod-PEG in case it was at its OFF state inside the cells. The probes were excited at $560 \mathrm{~nm}$ and the fluorescence was collected between 570 and $700 \mathrm{~nm}$ with the same setting for Rhod-2 and Rhod-PEG. The nucleus was stained with Hoechst ( $5 \mu \mathrm{g} / \mathrm{mL}$, blue color) and the plasma membrane with MemBright ${ }^{\circledR}-488$ (200 nM, green color).

\section{Monitoring $\mathrm{Ca}^{2+}$ transients in neurons}

Encouraged by the enhanced features of Rhod-PEG compared to Rhod-2, we compared their ability to measure $\mathrm{Ca}^{2+}$ transients in neurons. Indeed, hydrophilic calcium probes are more suitable for patch-clamp recording experiments in neuroscience where a glass pipette is filled with a solution containing a calcium probe that diffuses in the cytoplasm of the neuron along their thin axonal and dendritic processes. Pyramidal neurons were thus patched at the level of their soma with pipettes containing Rhod-PEG or Rhod-2 together with Alexa-488 dextran, to reveal the morphology of the cells (Figure 6A). We measured the Rhod-PEG or Rhod-2 basal fluorescence $\left(\mathrm{F}_{0}\right)$ and the fluorescence enhancement $\left(\Delta \mathrm{F} / \mathrm{F}_{0}\right)$ induced by bursts of action potentials (see Materials and Methods), at different distances from the soma and at different time points after starting the intracellular dye dialysis. Rhod-PEG allowed the detection of action potential-induced calcium transients with a high sensitivity: the obtained $\Delta \mathrm{F} / \mathrm{F}_{0}$ values often exceeded $100 \%$ (Figure $6 \mathrm{~A}$ ) and was on average as large or larger than those obtained with Rhod-2 (Figure 6B). High signal to noise ratios were obtained in the dendrites at relatively long distance from the soma $(50 \mu \mathrm{m})$. Interestingly, reliable calcium transients could be measured more rapidly in the dendrite of neurons with Rhod-PEG than with Rhod2 (Figure 6B, C), probably as a result of reduced non- 
specific interactions with intracellular membranes of the former probe. In line with this observation, the ratios of basal fluorescence between the dendrites and the soma ( $\mathrm{F}_{0}$ dendrite $/ \mathrm{F}_{0}$ soma) were higher (up to 2-fold) with RhodPEG than with Rhod-2 (Figure 6D). Identical observations were made when comparing Rhod-PEG and dextran-Alexa488 (Figure $\mathrm{S} 6$ ), the $\mathrm{F}_{0}$ dendrite $/ \mathrm{F}_{0}$ soma ratios were 0.37 and 0.76 respectively. Altogether, these results indicate that PEGylation leads to a faster and more efficient diffusion than Rhod-2 in the cytosol of thin neuronal dendrites and provide high sensitivity detection of cytosolic calcium fluctuations in these confined cellular processes.
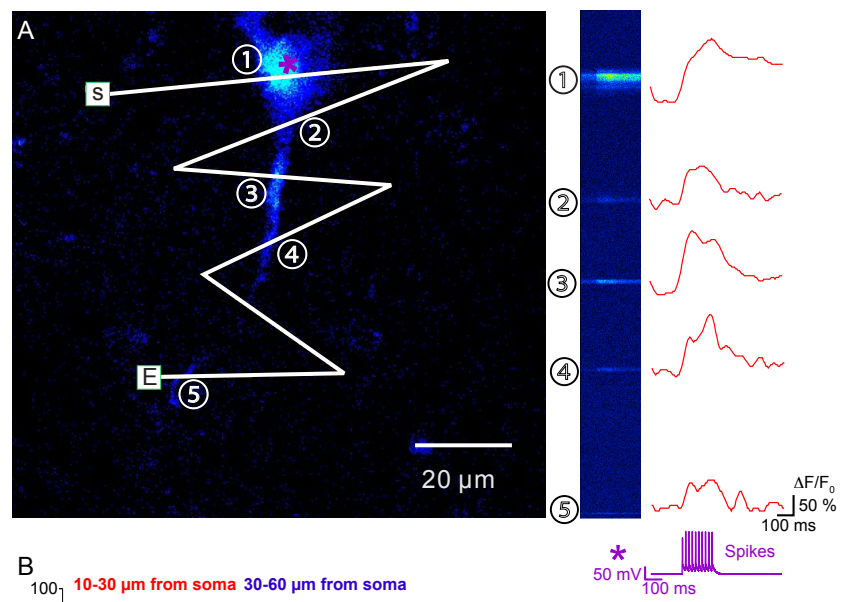

$\mathrm{B}$ 10-30 $\mu \mathrm{m}$ from soma $30-60 \mu \mathrm{m}$ from som $80 . \square \square$ Rhod-PEG $\square \square$ Rhod-2

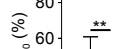

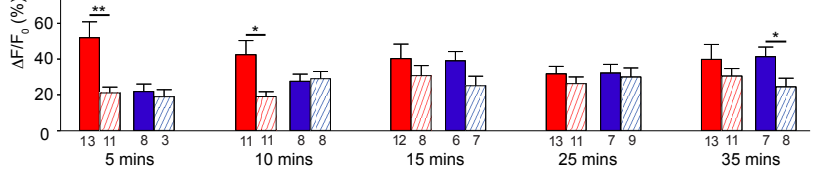

$\mathrm{C}_{100}, 30-60 \mu \mathrm{m}$ from soma
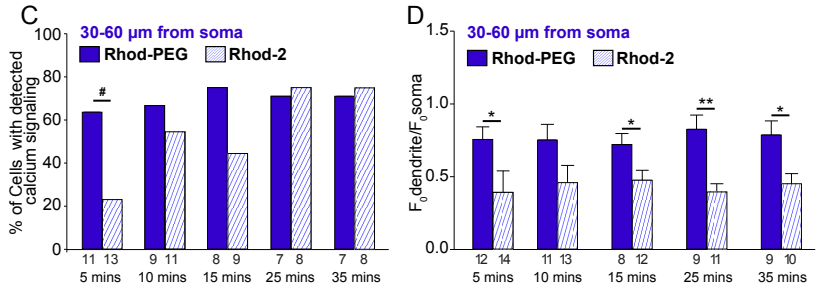

Figure 6. Comparison of the somatic and dendritic calcium signals measured with Rhod-2 and Rhod-PEG during trains of action potentials triggered in pyramidal cells of the mouse neocortex. (A), left: Two-photon imaging of a layer V neuron of the somatosensory cortex, labeled with Alexa-488. The white line indicates the trajectory of the line scan. Right: Representation of the RhodPEG florescence during the line scan. The vertical axis corresponds to the scanned position (as shown on the image on the left) and the horizontal axis represents time $(800 \mathrm{~ms})$. The change in Rhod-PEG florescence is color-coded. Numbers indicate ROIs. Red traces on the right represent the Rhod-PEG signals induced by a train of action potentials (lower trace) at specific somatic and dendritic locations indicated by the numbers. $\mathrm{S}$ and $\mathrm{E}$ indicate the starting point and the end position of the line scan. (B), Quantitative comparison of the Rhod-PEG and Rhod-2 signals at different time points after the beginning of the recordings at proximal and more distal dendritic locations. The numbers below the bars indicate the number of measurements. (C), Percentage of neurons loaded with Rhod-PEG or Rhod-2 in which a calcium response was detected in the $30-60 \mu \mathrm{m}$ distal part of the dendrites. (D), Comparison of the dendrite to soma ratio of the basal fluorescence for Rhod-PEG and Rhod-2 at different time points after the beginning of the intracellular dialysis. The numbers below the bars indicate the number of patched neurons. Error bars represent mean \pm S.E.M. ${ }^{*} \mathrm{P}<0.05, * * \mathrm{P}<0.01$. (Unpaired t-test). ${ }^{\#} \mathrm{P}<0.05$ (Chi squared test).

\section{CONCLUSION}

Newly developed Rhod-PEG combines the physicochemical advantages and reliability of molecular calcium probes with the hydrophilicity and stealth of the dextran conjugates. Indeed, the PEGylation of the fluorophore significantly reduced the non-specific interactions with membranes and mitochondria as well as enhanced the brightness and the fluorescence turn-on response of the $\mathrm{Ca}^{2+}$ probe. Consequently, Rhod-PEG, diffused more efficiently in neurons and in a faster way than its lipophilic version, Rhod-2. Additionally it was found to display higher sensitivity at short times after patching. In conclusion, RhodPEG is an improved alternative to the usual red-emitting calcium probes for sensing calcium transients in a reliable manner and at long distances from the neurons' soma.

\section{Materials and methods}

\section{Synthesis}

All starting materials for synthesis were purchased from Alfa Aesar, Sigma Aldrich or TCI Europe and used as received unless stated otherwise. NMR spectra were recorded on a Bruker Avance III $400 \mathrm{MHz}$ spectrometer. Mass spectra were obtained using an Agilent Q-TOF 6520 mass spectrometer.

\section{Spectroscopy}

Milli-Q water (Millipore) was used in all experiments. Absorption and emission spectra were recorded on a Cary 400 Scan ultraviolet-visible spectrophotometer (Varian) and a FluoroMax-4 spectrofluorometer (Horiba Jobin Yvon) equipped with a thermostated cell compartment, respectively. For standard recording of fluorescence spectra, the excitation wavelength was set to $530 \mathrm{~nm}$ and emission was recorded from 540 to $700 \mathrm{~nm}$. All the spectra were corrected from wavelength-dependent response of the detector. The fluorescence and absorption spectra of the corresponding blank suspension of lipid vesicles without the probe was subtracted from these spectra. The calcium buffer solutions ranging from 0 to $39 \mu \mathrm{M}$ were obtained from a calcium calibration buffer kit (ThermoFisher). Dioleoylphosphatidylcholine (DOPC), sphingomyelin (SM), and cholesterol $(\mathrm{Ch})$ were purchased from Sigma-Aldrich. Large unilamellar vesicles (LUVs) were obtained by the extrusion method as previously described. ${ }^{31}$ Briefly, a suspension of multilamellar vesicles was extruded by using a Lipex Biomembranes extruder (Vancouver, Canada). The size of the filters was first $0.2 \mu \mathrm{m}$ ( 7 passages) and 
thereafter $0.1 \mu \mathrm{m}$ (10 passages). This generates monodisperse LUVs with a mean diameter of $0.11 \mu \mathrm{m}$ as measured with a Malvern Zetasizer Nano ZSP (Malvern, U.K.). LUVs were labelled by adding $5 \mu \mathrm{L}$ of probe stock solution in dimethyl sulfoxide to $1 \mathrm{~mL}$ solutions of vesicles. A $20 \mathrm{mM}$ phosphate buffer, $\mathrm{pH} 7.4$, was used in these experiments. Molar ratios of probes to lipids were 1 to 200 .

\section{TPE measurements}

Two-photon absorption cross-section measurements were performed using Rhodamine B in methanol as a calibration standard according to the method of Webb et al. Two-photon excitation was provided by an InSight DS + laser (Spectra Physics) with a pulse duration of $120 \mathrm{fs}$. The laser was focused with an achromatic lens ( $\mathrm{f}=2 \mathrm{~cm}$ ) in a cuvette containing the dye $\left(2.3 \mu \mathrm{M}\right.$ for Rhod-PEG, $4.7 \mu \mathrm{M}$ for Rhod-2) in $1 \mathrm{mM} \mathrm{Ca}^{2+}$ MOPS (30 mM), $\mathrm{KCl}(100 \mathrm{mM}), \mathrm{pH} 7.2)$ and the spectra were recorded with a fibered spectrometer (Avantes) by collecting the fluorescence emission at $90^{\circ}$ with a $20 \times$ Olympus objective.

\section{Confocal imaging}

$\mathrm{KB}$ cells (ATCC® CCL-17) were grown in minimum essential medium (MEM, Gibco-Invitrogen) with $10 \%$ fetal bovine serum (FBS, Lonza), 1\% non-essential amino acids (GibcoInvitrogen), 1\% MEM vitamin solution (Gibco-Invitrogen), $1 \%$ L-Glutamine (Sigma Aldrich) and $0.1 \%$ antibiotic solution (gentamicin, Sigma-Aldrich) at $37^{\circ} \mathrm{C}$ in humidified atmosphere containing $5 \% \mathrm{CO}_{2}$. Cells were seeded onto a chambered coverglass (IBiDi $\AA$ ) at a density of $5 \times 10^{4}$ cells/well $24 \mathrm{~h}$ before the microscopy measurement. For imaging, the culture medium was removed and the attached cells were washed with Opti-MEM (Gibco-Invitrogen). Next, the cells were incubated in Opti-MEM with Hoechst $(5 \mu \mathrm{g} / \mathrm{mL})$ to stain the nuclei (blue colour) and in the presence of Rhod-2 $(1 \mu \mathrm{M})$ or Rhod-PEG (1 $\mu \mathrm{M})$ for 2 hours (red colour), the cells were washed two times with HBSS and visualized in HBSS. The plasma membrane was stained with MemBright ${ }^{\circledR}-488(200 \mathrm{nM})$ prior to imaging (green colour). To reach a concentration of ATP of $100 \mu \mathrm{M}$, $100 \mu \mathrm{L}$ of a $1.1 \mathrm{mM}$ solution of ATP was added to the $1 \mathrm{~mL}$ cell medium.

\section{Slice preparation}

All experiments were approved by the ethics committee of the University of Paris Descartes (registered numbers CEEA34.EA.027.11 and CEEA16-032) and followed guidelines of the European Union for the care and use of laboratory animals (Council directive 86/609EC). 3 to 4 weeks C57BL/6 wild-type mouse were anaesthetized with isoflurane and decapitated. Cortical slices $(300 \mu \mathrm{m})$ were prepared in an icecold solution containing (in $\mathrm{mM}$ ): $1 \mathrm{CaCl}_{2}$ and $5 \mathrm{MgCl}_{2}, 215$ Sucrose, $2.5 \mathrm{KCl}, 1.25 \mathrm{NaH}_{2} \mathrm{PO}_{4}, 26 \mathrm{NaHCO}_{3}, 20$ Glucose, 5 Sodium pyruvate, bubbled with $5 \% \mathrm{CO}_{2}$ and $95 \% \mathrm{O}_{2}, \mathrm{pH} 7.4$, $310 \mathrm{mOsm}$. After cutting, slices were immersed at $34^{\circ} \mathrm{C}$ for 30-40 mins and then maintained at room temperature for $0.5-4$ $\mathrm{h}$ in $5 \% \mathrm{CO}_{2}$ and $95 \% \mathrm{O}_{2}$ saturated artificial cerebrospinal fluid (aCSF) containing (in $\mathrm{mM}$ ): $1 \mathrm{MgCl}_{2}, 2 \mathrm{CaCl}_{2}, 126$
$\mathrm{NaCl}, 2.5 \mathrm{KCl}, 26 \mathrm{NaHCO}_{3}, 1.25 \mathrm{NaH}_{2} \mathrm{PO}_{4}, 20 \mathrm{D}$-glucose, and 1 Sodium pyruvate, $\mathrm{pH}$ 7.4, $310 \mathrm{mOsm}$.

\section{Electrophysiology}

Slices were immersed into a recording chamber perfused with $\operatorname{aCSF}\left(3 \mathrm{ml} / \mathrm{min}, 32^{\circ} \mathrm{C}\right)$. Whole-cell current-clamp recordings were performed in layer $\mathrm{V}$ pyramidal neurons of somatosensory cortex with glass pipettes (4-6 M) filled with an intracellular solution containing (in $\mathrm{mM}$ ): $10 \mathrm{KCl}, 0.5$ EGTA, $125 \mathrm{~K}$-Gluconate, $2 \mathrm{MgCl}_{2}, 10$ HEPES, $0.4 \mathrm{Na}^{+}$-GTP, 4 ATP-Na 2,10 Phosphocreatine disodium salt, 0.025 Alexa488 hydrazide sodium salt and 0.1 Rhod-2 or Rhod-PEG, $\mathrm{pH}$ 7.4, 280-290 mOsm. An Axopatch 200B (Molecular Devices) amplifier was used to perform patch-clamp recordings. Electronic signals were filtered at $5 \mathrm{KHz}$ and sampled at $10 \mathrm{KHz}$.

\section{Two-photon calcium imaging}

Fluorescence was imaged using a $40 \times$ water-immersion objective (Olympus) with a custom-built two-photon laserscanning microscope. Broken line scan acquisition $(\sim 100 \mathrm{~Hz}$, $0.8 \mathrm{~s}$, repeated 4 times with an interval $15 \mathrm{~s}$ ) with a custommade software (LabVIEW, National Instruments) was performed every 5 mins after establishing the whole-cell configuration to measure calcium signals in the soma and the dendrites of the recorded neurons. During calcium imaging acquisition, we triggered 10 action potentials (10 pulses of $2 \mathrm{~ms}$ duration at $50 \mathrm{~Hz}$ ) by injecting currents of appropriate amplitude through the patch pipette to induce calcium signals in soma and dendrites. Alexa-488 and Rhod-2/Rhod-PEG fluorophores were excited at $830 \mathrm{~nm}$, the two emission signals were separated by a dichroic $(560 \mathrm{~nm})$ and collected by two different photomultiplier tubes (PMT).

Calcium signals were analyzed in region of interest (ROI) covering the soma and dendrites during line scan imaging. For analysis, fluorescent signals were filtered at $5 \mathrm{~Hz}$ in Clampfit (Molecular Devices) and normalized changes in Rhod-2/RhodPEG fluorescence were calculated as $\Delta \mathrm{F} / \mathrm{F}_{0}=\left(\mathrm{F}-\mathrm{F}_{0}\right) / \mathrm{F}_{0}$. Calcium signals triggered by spike trains were identified by setting a threshold of 2 standard deviations of the baseline signal (i.e. before stimulation) and measured at their peak amplitude.

\section{ASSOCIATED CONTENT}

\section{Supporting Information}

Synthesis, protocols, characterizations and spectra are available in the supporting Information. The Supporting Information is available free of charge on the ACS Publications website.

\section{AUTHOR INFORMATION}

\section{Corresponding Author}

* Email address: mayeul.collot@unistra.fr

\section{Author Contributions}

The manuscript was written through contributions of all authors. All authors have given approval to the final version of the manuscript. $\ddagger$ These authors contributed equally. 


\section{ACKNOWLEDGMENT}

This work was supported by ERC Consolidator grant BrightSens 648528 and by grants from the Fondation pour la Recherche Médicale (FRM: DEQ20140329488) and the European Union (ERA-NET Neuron BrIE). We thank Tkhe Kyong FAM for the cell culture and Romain Vauchelles for his assistance at the PIQ imaging platform, Lydia Danglot for discussion, Serge Charpak for the access to the twophoton microscope and Yannick Goulam for his help with imaging experiments.

\section{REFERENCES}

(1) Clapham, D. E. Calcium Signaling. Cell 2007, 131 (6), 1047-1058.

(2) Brini, M.; Calì, T.; Ottolini, D.; Carafoli, E. Neuronal Calcium Signaling: Function and Dysfunction. Cell. Mol. Life Sci. 2014, 71 (15), 2787-2814.

(3) Burgoyne, R. D.; Haynes, L. P. Sense and Specificity in Neuronal Calcium Signalling. Biochim. Biophys. Acta BBA Mol. Cell Res. 2015, 1853 (9), 1921-1932.

(4) Grienberger, C.; Konnerth, A. Imaging Calcium in Neurons. Neuron 2012, 73 (5), 862-885.

(5) Hamilton, G. R. C.; Sahoo, S. K.; Kamila, S.; Singh, N.; Kaur, N.; Hyland, B. W.; Callan, J. F. Optical Probes for the Detection of Protons, and Alkali and Alkaline Earth Metal Cations. Chem. Soc. Rev. 2015, 44 (13), 4415-4432.

(6) Yin, J.; Hu, Y.; Yoon, J. Fluorescent Probes and Bioimaging: Alkali Metals, Alkaline Earth Metals and PH. Chem. Soc. Rev. 2015, 44 (14), 4619-4644.

(7) Grynkiewicz, G.; Poenie, M.; Tsien, R. Y. A New Generation of $\mathrm{Ca} 2+$ Indicators with Greatly Improved Fluorescence Properties. J. Biol. Chem. 1985, 260 (6), 3440-3450.

(8) Chen, T.-W.; Wardill, T. J.; Sun, Y.; Pulver, S. R.; Renninger, S. L.; Baohan, A.; Schreiter, E. R.; Kerr, R. A.; Orger, M. B.; Jayaraman, V.; Looger, L. L.; Svoboda, K.; Kim, D. S. Ultrasensitive Fluorescent Proteins for Imaging Neuronal Activity. Nature 2013, 499 (7458), 295-300.

(9) Bonora, M.; Giorgi, C.; Bononi, A.; Marchi, S.; Patergnani, S.; Rimessi, A.; Rizzuto, R.; Pinton, P. Subcellular Calcium Measurements in Mammalian Cells Using Jellyfish Photoprotein Aequorin-Based Probes. Nat. Protoc. 2013, 8 (11), 2105-2118.

(10) Oheim, M.; van 't Hoff, M.; Feltz, A.; Zamaleeva, A.; Mallet, J.-M.; Collot, M. New Red-Fluorescent Calcium Indicators for Optogenetics, Photoactivation and Multi-Color Imaging. Biochim. Biophys. Acta-Mol. Cell Res. 2014, 843 (10), 2284-2306.

(11) Hirabayashi, K.; Hanaoka, K.; Egawa, T.; Kobayashi, C.; Takahashi, S.; Komatsu, T.; Ueno, T.; Terai, T.; Ikegaya, Y.; Nagano, T.; Urano, Y. Development of Practical Red Fluorescent Probe for Cytoplasmic Calcium Ions with Greatly Improved Cell-Membrane Permeability. Cell Calcium 2016, 60 (4), 256-265.

(12) Egawa, T.; Hanaoka, K.; Koide, Y.; Ujita, S.; Takahashi, N.; Ikegaya, Y.; Matsuki, N.; Terai, T.; Ueno, T.; Komatsu, T.; Nagano, T. Development of a Far-Red to Near-Infrared Fluorescence Probe for Calcium Ion and Its Application to Multicolor Neuronal Imaging. J. Am. Chem. Soc. 2011, 133 (36), 14157-14159.

(13) Collot, M.; Ponsot, F.; Klymchenko, A. S. Ca-NIR: A Ratiometric near-Infrared Calcium Probe Based on a Dihydroxanthene-Hemicyanine Fluorophore. Chem. Commun. 2017, 53 (45), 6117-6120.
Matsui, A.; Umezawa, K.; Shindo, Y.; Fujii, T.; Citterio, D.; Oka, K.; Suzuki, K. A Near-Infrared Fluorescent Calcium Probe: A New Tool for Intracellular Multicolour Ca2+ Imaging. Chem. Commun. 2011, 47 (37), 10407-10409.

(15) Life Technologies https://www.thermofisher.com/.

(16) Egawa, T.; Hirabayashi, K.; Koide, Y.; Kobayashi, C.; Takahashi, N.; Mineno, T.; Terai, T.; Ueno, T.; Komatsu, T.; Ikegaya, Y.; Matsuki, N.; Nagano, T.; Hanaoka, K. Red Fluorescent Probe for Monitoring the Dynamics of Cytoplasmic Calcium Ions. Angew. Chem. Int. Ed. 2013, 52 (14), 38743877.

(17) Luccardini, C.; Yakovlev, A. V.; Pasche, M.; Gaillard, S.; Li, D.; Rousseau, F.; Ly, R.; Becherer, U.; Mallet, J.-M.; Feltz, A.; Oheim, M. Measuring Mitochondrial and Cytoplasmic $\mathrm{Ca} 2+$ in EGFP Expressing Cells with a LowAffinity Calcium Ruby and Its Dextran Conjugate. Cell Calcium 2009, 45 (3), 275-283.

(18) Collot, M.; Loukou, C.; Yakovlev, A. V.; Wilms, C. D.; Li, D.; Evrard, A.; Zamaleeva, A.; Bourdieu, L.; Leger, J.-F.; Ropert, N.; Eilers, J.; Oheim, M.; Feltz, A.; Mallet, J.-M. Calcium Rubies: A Family of Red-Emitting Functionalizable Indicators Suitable for Two-Photon Ca2+ Imaging. J. Am. Chem. Soc. 2012, 134 (36), 14923-14931.

(19) Collot, M.; Wilms, C. D.; Bentkhayet, A.; Marcaggi, P.; Couchman, K.; Charpak, S.; Dieudonné, S.; Häusser, M.; Feltz, A.; Mallet, J.-M. CaRuby-Nano: A Novel High Affinity Calcium Probe for Dual Color Imaging. eLife 2015, 4, e05808.

(20) Zamaleeva, A. I.; Despras, G.; Luccardini, C.; Collot, M.; de Waard, M.; Oheim, M.; Mallet, J.-M.; Feltz, A. FRET-Based Nanobiosensors for Imaging Intracellular $\mathrm{Ca} 2+$ and $\mathrm{H}+\mathrm{Mi}-$ crodomains. Sensors 2015, 15 (9), 24662-24680.

(21) Ogawa, M.; Kosaka, N.; Choyke, P. L.; Kobayashi, H. HType Dimer Formation of Fluorophores: A Mechanism for Activatable, in Vivo Optical Molecular Imaging. ACS Chem. Biol. 2009, 4 (7), 535-546.

(22) Despras, G.; Zamaleeva, A. I.; Dardevet, L.; Tisseyre, C.; Magalhaes, J. G.; Garner, C.; Waard, M. D.; Amigorena, S.; Feltz, A.; Mallet, J.-M.; Collot, M. H-Rubies, a New Family of Red Emitting Fluorescent PH Sensors for Living Cells. Chem. Sci. 2015, 6 (10), 5928-5937.

(23) Walkey, C. D.; Olsen, J. B.; Guo, H.; Emili, A.; Chan, W. C W. Nanoparticle Size and Surface Chemistry Determine Serum Protein Adsorption and Macrophage Uptake. J. Am. Chem. Soc. 2012, 134 (4), 2139-2147.

(24) Jokerst, J. V.; Lobovkina, T.; Zare, R. N.; Gambhir, S. S. Nanoparticle PEGylation for Imaging and Therapy. Nanomed. 2011, 6 (4), 715-728.

(25) Karpenko, I. A.; Collot, M.; Richert, L.; Valencia, C.; Villa, P.; Mély, Y.; Hibert, M.; Bonnet, D.; Klymchenko, A. S. Fluorogenic Squaraine Dimers with Polarity-Sensitive Folding As Bright Far-Red Probes for Background-Free Bioimaging. J. Am. Chem. Soc. 2015, 137 (1), 405-412.

(26) Karpenko, I. A.; Kreder, R.; Valencia, C.; Villa, P.; Mendre, C.; Mouillac, B.; Mely, Y.; Hibert, M.; Bonnet, D.; Klymchenko, A. S. Red Fluorescent Turn-On Ligands for Imaging and Quantifying G Protein-Coupled Receptors in Living Cells. Chembiochem 2014, 15 (3), 359-363.

(27) Duan, W.; Wei, H.; Cui, T.; Gao, B. A Membrane Permeable Fluorescent $\mathrm{Ca} 2+$ Probe Based on Bis-BODIPY with Branched PEG. J. Mater. Chem. B 2015, 3 (5), 894-898.

(28) Minta, A.; Kao, J. P.; Tsien, R. Y. Fluorescent Indicators for Cytosolic Calcium Based on Rhodamine and Fluorescein Chromophores. J. Biol. Chem. 1989, 264 (14), 8171-8178.

(29) Magde, D.; Rojas, G. E.; Seybold, P. G. Solvent Dependence of the Fluorescence Lifetimes of Xanthene Dyes. Photochem. Photobiol. 1999, 70 (5), 737-744. 
(30) Kantner, K.; Ashraf, S.; Carregal-Romero, S.; CarrilloCarrion, C.; Collot, M.; del Pino, P.; Heimbrodt, W.; De Aberasturi, D. J.; Kaiser, U.; Kazakova, L. I.; Lelle, M.; de Baroja, N. M.; Montenegro, J. M.; Nazarenus, M.; Pelaz, B.; Peneva, K.; Gil, P. R.; Sabir, N.; Schneider, L. M.; Shabarchina, L. I.; Sukhorukov, G. B.; Vazquez, M.; Yang, F.; Parak, W. J. Particle-Based Optical Sensing of Intracellular Ions at the Example of Calcium - What Are the Experimental Pitfalls? Small 2015, 11 (8), 896-904.

(31) Hope, M. J.; Bally, M. B.; Webb, G.; Cullis, P. R. Production of Large Unilamellar Vesicles by a Rapid Extrusion Procedure: Characterization of Size Distribution, Trapped Volume and Ability to Maintain a Membrane Potential. Biochim. Biophys. Acta 1985, 812 (1), 55-65. 